\title{
Malaria y determinantes sociales de la salud: un nuevo marco heurístico desde la medicina social latinoamericana
}

\author{
Juan Gabriel Piñeros ${ }^{1,2}$ \\ 1 Grupo de Malaria, Universidad de Antioquia, Medellín, Colombia \\ 2 Doctorado Interfacultades en Salud Pública, Universidad Nacional de Colombia, Bogotá, Colombia.
}

Tradicionalmente, la investigación y el estudio de la malaria se han hecho bajo el paradigma positivista de la ciencia y su concepción biomédica de la enfermedad. Desde esta perspectiva se han diseñado las diversas acciones y estrategias para contrarrestarla. Sin embargo, a pesar de un siglo de experiencia científica, y de la profundidad y minuciosidad logradas en el campo de conocimiento de malaria, esto no se ha traducido en una disminución constante y progresiva de su carga epidemiológica.

Este ensayo plantea la necesidad de un cambio en la concepción de la malaria, configurándola como un proceso de carácter biológico y social, en el cual las posibilidades genotípicas y fenotípicas de la relación entre huésped y parásito y de la expresión clínica de la enfermedad, se articulen a las dinámicas históricas y sociales de los espacios donde se presentan.

Además, se propone replantear la investigación epidemiológica de esta entidad tomando como base la visualización del carácter dinámico, heterogéneo, dialéctico y complejo de las organizaciones biosociales que constituyen la realidad de la malaria, desde la estructura social hasta el nivel genético y fenotípico de los individuos parasitarios, vectoriales y humanos. Para esto se sugiere: 1) asumir el enfoque latinoamericano de factores determinantes sociales de la salud, 2) incorporar nuevas categorías analíticas (por ejemplo, territorio social de la malaria) y nuevas herramientas de investigación (matrices de procesos críticos de la determinación social), y 3) reinterpretar las categorías epidemiológicas convencionales de las enfermedades infecciosas, como la transmisión y la contagiosidad.

Palabras clave: malaria/transmisión, paludismo, epidemiología, medicina social, transmisión, investigación.

\begin{abstract}
Malaria and social health determinants: a new heuristic framework from the perspective of Latin American social medicine

Traditionally, malaria research and study have followed the positivist scientific paradigm and its biomedical conception of disease. From this perspective, diverse control actions and strategies have been designed. However, despite a century of scientific experience and the depth and thoroughness achieved in the knowledge of malaria, this has not been translated into a constant and progressive decrease of its epidemiological burden.

This essay argues for the need for a change in malaria conception, reconfiguring it as a process of biological and social character, where the geno-phenotypical possibilities of the host-parasite relationship and of the disease's clinical expression are articulated with the historic and social dynamics of the spaces in which they occur.

In addition, it proposes rethinking the epidemiological research of this entity on the basis of the visualization of the dynamic, heterogeneous, dialectic and complex character of biosocial organizations that constitute the reality of malaria (from the social structure to the genetic and phenotypic level of parasite individuals, vectors and humans). To achieve this, it is suggested that: 1) the Latin American perspective on the social determinants of health be adopted; 2) new analytical categories (for instance, malaria social territory) and new investigation tools (matrices of critical processes of social determination) be incorporated, and 3) the conventional epidemiological categories of infectious diseases such as the transmission and infectiousness be reinterpreted.
\end{abstract}

Key words: malaria/transmission, epidemiology, social medicine, transmission, research.

Correspondencia:

Juan Gabriel Piñeros, Sede de Investigación Universitaria, Calle 62 № 52-59, laboratorio 610-611, Medellín, Colombia. Telefax: (574) 2196487

malaria@arhuaco.udea.edu.co y jgpinerosj@unal.edu.co

Recibido: 09/03/09; aceptado: 09/12/09
Las acciones, estrategias y políticas diseñadas para proteger, mejorar o lograr la salud dependen de la concepción ontológica del objeto de estudio y del enfoque epistemológico en que se inscribe su investigación (1). 
Desde el descubrimiento de los corpúsculos de Laveran a finales del siglo XIX, en pleno surgimiento de la bacteriología y de la lógica unicausal, pasando por la mirada ecológica y multidisciplinaria de la medicina tropical, hasta el desentrañamiento de los textos nucleicos de Plasmodium falciparum y Anopheles gambie y las revelaciones, sorpresas, paradojas y contradicciones que acompañaron sus lecturas con el advenimiento actual de la genómica, la proteómica y la transcriptómica, la malaria se ha concebido como una realidad constituida fundamentalmente por elementos de naturaleza biológica (parásito, vector, humano, genes, proteínas, etc.).

Su estudio e investigación se ha hecho bajo las miradas mecánicas de los paradigmas positivista y neopositivista, privilegiando la descripción minuciosa, fragmentada, reducida y matemática de la realidad, y las relaciones lineales (causaefecto) entre la maraña de elementos, organismos y situaciones para explicarla (2). Incluso, cuando se ha ampliado el campo de observación para incluir los ámbitos sociales, económicos, políticos y culturales de los contextos donde se produce la enfermedad o que caracterizan a los sujetos que la padecen, el énfasis se ha puesto en establecer relaciones de causalidad entre aspectos de las condiciones materiales de vida, la posición social de los individuos y algunos indicadores técnicos que reflejan parte de la realidad social (índice de pobreza, índice de calidad de vida, índice de calidad de la vivienda, entre otros), con los fenómenos palúdicos.

No obstante el amplio conocimiento científico producido por las ciencias biomédicas y la salud pública, y los incalculables avances técnicos y tecnológicos producidos a partir de esta forma de entender la malaria, ésta continúa presentando frecuencias alarmantes. Al día de hoy, no se ha modificado significativamente la tendencia histórica de la enfermedad, ni se ha logrado mitigar los efectos negativos sobre las poblaciones vulnerables, como niños, mujeres y adultos trabajadores dedicados a actividades económicas en las zonas rurales de los países endémicos.

Anualmente se reportan entre 250 y 500 millones de casos y entre 800.000 y tres millones de defunciones por malaria en el mundo, principalmente en las regiones más pobres y con mayor exclusión social (3). A esto se suman fenómenos como la resistencia de Plasmodium spp. a los antipalúdicos, la resistencia de Anopheles spp. a los insecticidas, el cambio climático mundial, la expansión mundial de las áreas geográficas con condiciones ecológicas propicias para la transmisión del agente infeccioso y el aumento de los movimientos masivos y globales de la población, todo lo cual contribuye a empeorar su panorama epidemiológico (4).

Lo anterior refleja una gran contradicción ya que, a pesar de los indiscutibles progresos en el conocimiento y la tecnología, éstos no se traducen en transformaciones reales de las condiciones de vida y de salud de las colectividades. Esta contradicción hace parte de lo que se ha denominado como "la crisis profunda de la salud pública" (5), cuyas raíces se ubican en el núcleo de los paradigmas científicos hegemónicos y sus formas de entender y conceptualizar los objetos en salud, tal como lo han señalado, a todo lo largo y ancho de América Latina, investigadores del movimiento de la medicina social, como Jaime Breilh (Ecuador), Asa Crisitina Larurell (México), Naomar Almeida Filho (Brasil) y Saúl Franco (Colombia), entre otros (6).

Ellos proponen que la salud tiene un carácter holístico, dinámico y complejo, en el que confluyen y se articulan en toda su esencia realidades biológicas y sociales. Además, han construido nuevos marcos heurísticos para entender, investigar y estudiar la salud y sus problemas, que involucran enfoques científicos alternativos para visualizar la complejidad de las relaciones sociales e históricas no lineales entre realidades en constante movimiento y de distinta naturaleza (7).

En el caso de la malaria, lo anterior implica asumir un enfoque de investigación y unas categorías que evidencien las relaciones sociales, que crean las posibilidades de su existencia en lugares y momentos concretos, y expliciten las dinámicas que median sus procesos epidemiológicos, y que determinan y explican las expresiones genéticas y fenotípicas de los individuos que la conforman (8).

El objetivo de este ensayo es presentar brevemente el modelo teórico de los factores sociales determinantes, construido por la medicina social latinoamericana, y proponer algunas categorías y herramientas metodológicas como alternativas para la investigación crítica de la malaria.

\section{Determinantes sociales de la salud: un enfoque alternativo para comprender la malaria}

La construcción de un enfoque alternativo para el abordaje de la salud y la enfermedad partió de la reflexión de los planos ontológico, epistemológico 
y metodológico de la salud pública como campo de conocimientos. En un marco crítico de casi cuarenta años, académicos latinoamericanos agrupados en el movimiento de medicina social, llamado en Brasil como "salud colectiva", han cuestionado la construcción de la salud como objeto de conocimiento hecha por la ciencia moderna, la cual se ha caracterizado por fragmentar la realidad en dicotomías como: biológico-social, sujeto-objeto, parte-totalidad y naturaleza-cultura, entre otras, por congelar el tiempo y la materia, y por negar implícitamente el carácter histórico de los procesos y los constantes movimientos de unión o separación y de reproducción o generación que existen entre sus elementos, desconociendo la profunda articulación de sus dimensiones en la medida en que comparten los mismos espacios y cuerpos y la dinámica de sus elementos que constantemente configuran totalidades y fragmentos (9).

A partir de estas premisas, se ha reconocido la naturaleza social de salud-enfermedad como parte de una misma realidad compleja, en la cual procesos particulares, como la malaria, se generan y reproducen en los procesos generales histórico -sociales, sus realidades y dinámicas diversas y localizadas, y en la interacción de procesos de determinación social organizados jerárquicamente $(1,9)$.

Con un enfoque materialista de la historia, se asume que las relaciones entre los grupos y los sujetos sociales, y de éstos con la naturaleza, determinan las formas históricas de enfermar y de morir. La salud es una constante unidad de cambio, expresión en la corporeidad humana y colectiva, del modo específico de apropiación de los recursos naturales y humanos bajo una determinada forma de organización social, y no sólo hechos aislados en razón de factores al azar que permitían la aparición de una enfermedad en un individuo particular. La salud o la enfermedad es el resultado del complejo proceso de producción o reproducción sociales, propiedad fundamental de las relaciones históricas de los seres humanos (10).

Entre los componentes y las dinámicas de cada uno de los dominios de la organización social, de acuerdo con su respectivo ámbito (social, económico, político, ambiental, cultural), y entre los que existen en distintos dominios, se presenta una constante dialéctica de poder que limita, permite y transforma la realidad, definiendo los procesos peligrosos y protectores que distribuyen la enfermedad y el bienestar en espacios (macro- micro) y sujetos (colectivos-individuales), y determinan las expresiones genotípica y fenotípica de la vida (humana, microbiológica, vectorial, etc.).

Para sortear esta complejidad, la epidemiología crítica se ha constituido en una herramienta que explica la distribución y frecuencia de saludenfermedad a partir de los procesos sociales e históricos de determinación, que se reflejan en una materialidad concreta y en una producción o reproducción sociales específicas, en cada espacio, sujeto y dimensión de la realidad (1).

En el dominio general, en el que se concentra la mayor fuerza de determinación, se localizan las lógicas históricas de orden político, económico, ideológico y cultural que conforman la estructura social y definen el modelo de acumulación de capital en un momento preciso (1). Estas lógicas explican los procesos y los ritmos de producción, los tipos de desarrollo de las fuerzas productivas, las relaciones de producción específicas, la distribución de la riqueza social y las formas de organización económica y social de los grupos y sujetos que conforman la sociedad.

Un segundo espacio, el dominio intermedio, comprende los procesos relacionales de los grupos sociales, los cuales son limitados por las lógicas estructurales y explican las posibilidades del modo de vida de los grupos, cuya reproducción social es el intercambio orgánico continuo entre los sujetos sociales, y de éstos con la naturaleza, de acuerdo con sus condiciones de clase social, género y etnia. Sus componentes incluyen las condiciones grupales de trabajo, la calidad y capacidad de disfrute de los bienes y servicios de consumo, la capacidad de construcción de soporte de organización que permita la producción de sujetos políticos, la relación con el ambiente, la capacidad de reproducción, creación y el ejercicio de relaciones culturales. Este proceso tiene sus particularidades según el espacio o territorio geográfico en que ocurre y su dinámica busca la transformación constante de las estructuras establecidas (1).

Por último, el dominio de los procesos singulares en el que se construyen los estilos de vida de las familias y los individuos -cuya posibilidad es el ejercicio del libre albedrío limitado por los dominios de factores determinación superiores, es decir, las posibilidades de vivir la vida que son permitidas por la forma en que estructuras sociales, económicas, políticas y culturales- se reproducen en los grupos y éstos, a su vez, en los sujetos. En este dominio se localizan aspectos de la cotidianidad social como 
los itinerarios personales y familiares en ámbitos como el trabajo y el consumo, las valoraciones y concepciones frente a la salud o la enfermedad y su cuidado, la capacidad individual de generar prácticas organizadas en salud y la relación inmediata con el entorno (1).

La realidad social así organizada y su constante movimiento de generación-reproducción, crean la necesidad de una racionalidad diferente para comprender los entramados complejos que explican los perfiles epidemiológicos, entre ellos, los de la malaria (1). Así, se retoma la dialéctica como racionalidad y se obliga a trazar caminos novedosos en la construcción de enfoques epistemológicos y metodológicos integrales en el estudio e investigación en salud pública, que involucren con urgencia miradas interdisciplinarias y transdisciplinarias, y que integren narrativas distintas en los modelos epidemiológicos.

El mayor cuerpo de producción teórica necesario para las deconstrucciones ontológica, epistemológica y metodológica que han generado una nueva forma de comprender el proceso de salud-enfermedad (11-14), se ha generado en América Latina. Sin embargo, las concepciones generadas bajo este nuevo marco epistémico no son exclusivas de la academia latinoamericana.

En los países llamados centrales, aunque el reconocimiento de la influencia de la realidad social sobre los problemas de salud data de mediados del siglo XIX, con la introducción del concepto de medicina social por Guerin y Virchow (15), la idea de que los entornos sociales y las formas de vivir de los individuos afectaban la salud no se divulgó ampliamente sino hasta mediados de los setenta, cuando Marc Lalonde, en su texto "Una nueva perspectiva en la salud de los canadienses", de 1974 (16), incluyó estos aspectos como puntos centrales de los estudios, políticas e intervenciones en salud pública que, luego, constituyeron la base de la declaración de Alma Ata de 1978 y de la política mundial de atención primaria en salud (17). Sin embargo, aunque los aspectos sociales, económicos, políticos y culturales desde este momento se han considerado en la explicación de la frecuencia y distribución de las enfermedades, y aunque durante la última década se ha desarrollado toda una rama de la epidemiología con enfoque ecosistémico para incluirlos dentro del análisis epidemiológico (18), no se puede decir que esto haya significado un cambio paradigmático. Al contrario, puede decirse que todo este desarrollo, producido en el seno de la epidemiología clásica, ha contribuido a la invisibilidad de la complejidad social de salud-enfermedad, dados los obstáculos epistemológicos propios de disciplina que ha construido miradas unívocas de la realidad.

No es en vano el reciente llamado de atención del reconocido investigador español Vicente Navarro, quien afirma que a pesar del gran interés mundial en estos aspectos, que ha llevado a conformar en el seno mismo de la Organización Mundial de la Salud la Comisión Mundial de Determinantes Sociales de la Salud, la mirada sigue siendo superficial, centrada en las consecuencias y no en las causas profundas e históricas de los problemas y desigualdades en salud que, necesariamente, implican enfoques teóricos alternativos (19).

\section{Territorio social de la malaria}

En el marco de la determinación social, es necesario tener en cuenta que la realidad general se matiza y especifica cuando se reproduce en el plano particular, el cual depende, sin lugar a dudas, del lugar donde se producen y reproducen los procesos de la sociedad. Es importante recalcar esta cualidad, dado que los procesos de salud-enfermedad están sujetos de manera determinante tanto al momento histórico como a la especialidad en el que se presentan. Esta relación es sustancialmente importante desde la construcción epidemiológica crítica, pues aunque una enfermedad tenga frecuencias y distribución similares entre la población de territorios diferentes, sus procesos explicativos son distintos y, también, lo son sus posibilidades de intervención.

Es importante considerar el concepto de territorio social, en la medida en que en su contenido se articula con los determinantes sociales de la salud. A diferencia de la geografía clásica, que ha estudiado y descrito el espacio físico tal como se observa, estático, la geografía humana o social ha consolidado en los últimos años un cuerpo teórico y metodológico para entender el espacio como producto de las dinámicas históricas, donde los sujetos se relacionan entre sí, del cual se adueñan, con el cual crean identidad y se reproducen socialmente: el territorio (20).

Según geógrafos, como Milton Santos, el territorio social articula en un entramado complejo la realidad material (natural o fabricada) con las dinámicas sociales contenidas en su interior y por fuera de ella, que definen las relaciones de los grupos y sujetos con el entorno. Así, es 
constituido por elementos fijos y por flujos, en una unidad que conjuga materialidad y acciones humanas. Estas últimas, conscientemente o no, son generadas por las relaciones de poder entre los órdenes económico, político, ideológico y cultural o sus posibles combinaciones, en el ejercicio de una lógica estructural específica que produce y reproduce la sociedad. El territorio social es una construcción histórica precisa (20).

En el caso específico de la malaria, el espacio tiene de por sí una particularidad importante en su complejidad comprensiva, dado que esta enfermedad se presenta en territorios sociales específicos y concretos con dinámicas históricas y sociales únicas, en un paisaje definido en continua transformación: el territorio rural de las zonas tropicales y la constante construcción o deconstrucción de su ruralidad.

En el contexto de análisis del enfoque latinoamericano de los determinantes sociales de la salud, el espacio concebido sólo como nicho ecológico natural de la enfermedad, se convierte con toda su complejidad natural en el territorio que se transforma por las lógicas históricas y sociales de cada dominio de determinación. De esta forma, la homogeneidad natural se trasforma en la heterogeneidad social que es necesaria para comprender la ubicación y la distribución de la enfermedad y su proceso de transmisión (21). Un ejemplo de lo anterior se puede evidenciar en los trabajos de Sabroza sobre dengue en Brasil, los cuales, a partir de la caracterización de los territorios sociales urbanos, explicaron los fenómenos de infestación de Aedes aegypti $(22,23)$.

El cuadro 1 resume la propuesta de una matriz de procesos críticos para el caso de la malaria, en la cual se presentan los principales componentes de cada dominio social, cuya dialéctica constituye un posible marco heurístico para este proceso.

\section{Transformación de las categorías epidemiológicas para comprender el proceso social de malaria}

Para el caso de malaria y de otras enfermedades transmitidas por vectores, la epidemiología crítica

Cuadro 1. Matriz de procesos críticos de la determinación social de malaria.

\begin{tabular}{|c|c|c|c|c|}
\hline \multicolumn{3}{|l|}{ Dimensión colectiva } & \multicolumn{2}{|l|}{ Dimensión individual } \\
\hline $\begin{array}{l}\text { Relaciones } \\
\text { estructurales } \\
\text { generales }\end{array}$ & $\begin{array}{l}\text { Lógica estructurales } \\
\text { en contradicción }\end{array}$ & $\begin{array}{l}\text { Modos de vida } \\
\text { (clase social, } \\
\text { sexo y etnia) } \\
\text { Patrones de } \\
\text { exposición colectivas } \\
\text { por espacio social }\end{array}$ & $\begin{array}{l}\text { Estilos de vida } \\
\text { (familiares e } \\
\text { individuales) } \\
\text { Patrones de } \\
\text { exposición individual }\end{array}$ & $\begin{array}{l}\text { Condiciones } \\
\text { genotípicas/fenotípicas }\end{array}$ \\
\hline $\begin{array}{l}\text { Económicas: } \\
\text { sistema y ritmos de } \\
\text { acumulación de capital } \\
\text { en zonas rurales }\end{array}$ & $\begin{array}{l}\text { Producción depredadora } \\
\text { y trabajo explotador o } \\
\text { producción sostenible } \\
\text { y justa }\end{array}$ & $\begin{array}{l}\text {-Prácticas de producción } \\
\text {-Características del } \\
\text { consumo simple } \\
\text { y ampliado } \\
\text {-Infraestructura física }\end{array}$ & $\begin{array}{l}\text {-Itinerario laboral } \\
\text { •Prácticas de } \\
\text { reposición de la } \\
\text { energía }\end{array}$ & $\begin{array}{l}\text { de los genotipos } \\
\text { circulantes } \\
\text {-Resistencia a los } \\
\text { antipalúdicos } \\
\text { Personas } \\
\text {-Inmunidad adquirida }\end{array}$ \\
\hline $\begin{array}{l}\text { Políticas: } \\
\text { ejercicio político y } \\
\text { primacía ideológica } \\
\text { del estado nación y } \\
\text { el Estado-región }\end{array}$ & $\begin{array}{l}\text { Ejercicio político de } \\
\text { homogenización limitado } \\
\text { y centrado en lo } \\
\text { económico o democracia } \\
\text { abierta, ejercicio activo de } \\
\text { la ciudadanía y centrado } \\
\text { en derechos humanos }\end{array}$ & $\begin{array}{l}\text {-Consolidación del sujeto } \\
\text { político y su integración } \\
\text { en las políticas estatales } \\
\text { (incluida salud) }\end{array}$ & $\begin{array}{l}\text {-Capacidad y barreras } \\
\text { al ejercicio del derecho } \\
\text { a la salud } \\
\text {-Planeación y ejecución } \\
\text { de acciones } \\
\text { transformadoras }\end{array}$ & $\begin{array}{l}\text { frente a la malaria } \\
\text {-Condiciones de protec- } \\
\text { ción o de peligro } \\
\text { frente a la infección } \\
\text { (por ejemplo, hemoglobi- } \\
\text { nopatías, desnutrición } \\
\text { crónica) } \\
\text {-Respuesta inflamatoria }\end{array}$ \\
\hline $\begin{array}{l}\text { Culturales: } \\
\text { diversidad étnica e } \\
\text { intercambio cultural }\end{array}$ & $\begin{array}{l}\text { Culturización o } \\
\text { reconocimiento de } \\
\text { la diversidad cultural }\end{array}$ & $\begin{array}{l}\text {-Significados, actitudes } \\
\text { y prácticas de salud }\end{array}$ & $\begin{array}{l}\text {-Valoración y } \\
\text { sentimientos } \\
\text { frente a la salud } \\
\text {-Prácticas de higiene } \\
\text { y alimentación }\end{array}$ & $\begin{array}{l}\text { a la infección } \\
\text {-Clínica } \\
\text { Vectores } \\
\text {-Caracterización y } \\
\text { distribución de los } \\
\text { criaderos de Anopheles }\end{array}$ \\
\hline Ambientales & $\begin{array}{l}\text { Explotación exagerada } \\
\text { del ecosistema } \\
\text { o manejo ambiental } \\
\text { sostenible }\end{array}$ & $\begin{array}{l}\text { - Transformación de } \\
\text { los entornos por las } \\
\text { actividades de los } \\
\text { grupos humanos }\end{array}$ & $\begin{array}{l}\text {-Valoración, tipo de } \\
\text { relación, prácticas } \\
\text { de disfrute y cuidado } \\
\text { del entorno ecológico } \\
\text { familiar }\end{array}$ & $\begin{array}{l}\text {-Comportamiento } \\
\text { entomológico } \\
\text { •Resistencia a los } \\
\text { insecticidas }\end{array}$ \\
\hline
\end{tabular}


debe reconstruir los marcos analíticos y narrativos tradicionales para poder describir las frecuencias, las distribuciones, los aspectos explicativos y las problemáticas asociadas, desde este enfoque de determinación social en salud.

Para lograr esto, es fundamental reescribir las categorías utilizadas por la epidemiología clásica para explicar las enfermedades infecciosas, como la transmisión y la contagiosidad (24), y pasar de concebirlas de forma estática y simple, consecuencias de relaciones lineales entre elementos particulares, a considerarlas como procesos históricos, complejos, con contradicciones y múltiples dimensiones, productos de una dialéctica constante entre fuerzas y acciones sociales que se reflejan en procesos y materialidades específicas (1). Esto significa entender que, desde las lógicas que rigen las relaciones sociales más generales, se generan procesos que influyen sobre las dinámicas de las comunidades y los sujetos, y que éstas se localizan en distintos espacios (los espacios domésticos, laborales, comunitarios, religiosos, etc.) que pueden ser caracterizados, en los que se desarrollan las actividades cotidianas para lograr la existencia biológica y social. Estos elementos permiten entender los patrones diferenciales de exposición y de vulnerabilidad entre los grupos y sujetos sociales.

\section{Proceso de transmisión}

La primera de estas categorías, la transmisión, pasaría de ser la simple movilización de un agente infeccioso de un huésped infectado a otro no infectado con la participación de un agente vector, a concebirse como el proceso generado por la dinámica entre la construcción de contextos infectivos específicos, que surgen de la interacción de los territorios sociales con la historia epidemiológica de la enfermedad y la construcción de los patrones de exposición posibles, que se deben a los modos y los estilos de vida que se observan y materializan en los espacios (10).

Las construcciones de los contextos infecciosos tratan de comprender la historia general del territorio y de la epidemiología local de la malaria, en aras de encontrar relaciones entre las dos. Con ayuda del diálogo entre la geografía, la historia y la epidemiología, se describiría la historia política, socioeconómica y ambiental de la zona palúdica, buscando identificar la lógica estructural predominante que dinamiza la región, localizar los territorios sociales que se conforman bajo estas lógicas, su distribución y tipo (comunitario, laboral, habitados por tal o cual grupo cultural, etc.), identificar los ecosistemas (bosques, selva húmeda, manglares, etc.) y sistemas productivos (agroindustrial, agricultura artesanal, pecuarios, explotación de minerales 0 de recursos no renovables) y determinar las formas en que históricamente los primeros han sido transformados o conservados por el hombre y los segundos han sido implementados y son manejados por ellos.

Respecto a las historias epidemiológicas de la malaria, el énfasis estaría dirigido a entender:

1) los patrones de distribución, ritmos y desplazamientos espaciales de la endemia de acuerdo con los agentes infecciosos, $P$. vivax, $P$. falciparum, $P$. malariae o $P$. ovale;

2) la ubicación y caracterización de los criaderos vectoriales más importantes;

3) la distribución, ritmos y dinámica de las poblaciones de las especies de Anopheles existentes en la zona;

4) las particularidades históricas de la endemia, tales como el grado de respuesta antipalúdica de los pacientes, la adaptabilidad de los anofeles asociada a los insecticidas, la circulación de genotipos del parásito con mayor asociación a morbilidad grave o a resistencia a medicamentos, o la circulación de fenotipos especiales como el asociado a la malaria placentaria, y

5) las acciones históricas emprendidas para contrarrestar la enfermedad, el lugar donde fueron realizadas, su periodicidad y su eficacia.

La construcción de los patrones de exposición requiere, para las enfermedades transmitidas por vectores como la malaria, construir la exposición a fuentes de agentes infecciosos (los enfermos) y las fuentes de agentes transmisores (los vectores). Estos patrones se generan para cada espacio social a partir de las dinámicas y las lógicas presentes en éste, y se materializan de acuerdo con los modos y los estilos de vida ejercidos por los grupos y sujetos según sus condiciones de clase social, género y etnia, que los exponen diferencialmente a las distintas fuentes de infección y transmisión. Para esta construcción, es necesario indagar sobre las distintas acciones o actividades que se realizan en cada espacio y responder a las preguntas: ¿quiénes?, ¿cuándo?, ¿dónde?, ¿cómo?, ¿por cuánto tiempo se realizan?, ¿cuáles interacciones entre los grupos sociales las explican? y ¿cuáles lógicas las determinan? (1). 
Así, si se trata de un espacio de tipo laboral, habrá que determinar, además de sus características materiales generales, los tipos de trabajo que ejercen los hombres, las mujeres, los operarios, los directivos, los mestizos, los indígenas, los afrodescendientes, los niños, los jóvenes y los ancianos, así como sus itinerarios, frecuencias, lugares en que realizan cada tarea específica y la duración de cada una de éstas. Además, se debe caracterizar la infraestructura existente, especificando si ésta está destinada a alojar a los trabajadores durante las horas de mayor transmisión de malaria, en caso de aquéllos con horarios nocturnos o que duermen en su lugar de trabajo o, por el contrario, determinar las características del tránsito de los trabajadores desde sus hogares al espacio laboral, en cuanto a la ruta, el medio de transporte utilizado, el tiempo invertido y el horario. Se debe tener en cuenta que en las zonas palúdicas es muy común el trabajo polivalente y esta indagación debe realizarse para cada uno de los distintos espacios de trabajo de cada sujeto, tratando de abarcar la gran complejidad del trabajo y la exposición a la malaria.

En el caso de los espacios comunitarios, es indispensable comprender las actividades realizadas por los miembros de la comunidad, teniendo en cuenta su tipo (productiva, de acción sobre el paisaje, deportiva, recreativa, religiosa o cultural), sus horarios, frecuencias, lugares de ejecución, participantes de acuerdo con su edad, género y etnia, así como los grados y tipos de transformación del entorno comunitario debido a éstas.

Para los espacios domiciliarios, es importante determinar las actividades que se realizan en dicho espacio, sobre todo aquellas que hacen referencia al trabajo doméstico, ya que éstas implican una diferencia marcada de los procesos sociales de acuerdo con el sexo. Esta indagación debe incluir una descripción pormenorizada de las distintas actividades ejercidas, sus lugares e itinerarios, y las relaciones de éstas con los entornos naturales y con las dinámicas sociales y comunitarias.

Con la información anterior, se definirá el grado de exposición a los enfermos y a los vectores mediante la indagación de:

1) distribución de enfermos en el espacio social;

2) distancia entre los enfermos y los no enfermos, principalmente en el período entre las 6 de la tarde y las 6 de la mañana (directa: duermen en la misma habitación o en la misma casa; próxima: en la misma cuadra; cercana: en la misma localidad o barrio; lejana: en otra localidad o barrio);

3) frecuencia y duración de la convivencia en el mismo período entre los no enfermos y los enfermos (permanente: todas las noches $y$ durante toda la noche; frecuente: todas las noches y durante algunos momentos de la noche; eventual: semanal);

4) ubicación, distribución, tipo (permanente o temporal, natural o debido a la acción humana) y características de los criaderos existentes en cada espacio;

5) distancia de los criaderos a las viviendas o lugares de habitación;

6) lugar, grado y duración de la exposición al mosquito (frecuencia de captura de mosquitos por especie en el interior del domicilio y en el peridomicilio, tasa de picaduras infectivas por especie de Anopheles, en los espacios anteriores, momento de mayor actividad vectorial y su duración);

7) presencia, tipo, material y calidad de las medidas de protección en las viviendas, y

8) presencia, tipo y calidad de las medidas de protección individual contra los vectores (toldillos, repelente, otros). En estas dos últimas, hay que tener en cuenta si son suministradas por el empleador cuando se trata del espacio laboral.

Por último, se deberá considerar el grado de capacitación o información de la comunidad respecto a la malaria y las acciones diseñadas para enfrentarla o evitarla (ausente, incompleto y de mala calidad, amplio y superficial, amplio y profundo), teniendo en cuenta las particularidades sociales y culturales de los distintos saberes y su coherencia con las prácticas. Y de igual forma, conocer los procesos que disminuyan o aumenten la vulnerabilidad en cuanto a la exposición, como la presencia de embarazadas, las cuales según algunos estudios, atraen con mayor frecuencia vectores, o el consumo de ciertos alimentos y sustancias como la vitamina $B$ que disminuyen la atracción del vector, entre otros (1).

\section{Proceso de contagiosidad}

La segunda categoría, la contagiosidad, es un concepto que parte de la posibilidad de un huésped o grupo de ellos de infectar a otro. En el caso de la 
malaria, consiste en la posibilidad de traspasar los agentes infecciosos del enfermo al mosquito; se relaciona con una mayor o menor capacidad de los grupos para mantener y permitir la reproducción de los parásitos en el interior del huésped humano (susceptibilidad) y con la duración de la infección.

Un reescritura de esta categoría deberá tener en cuenta que la contagiosidad ante todo es producto del contexto histórico y social en el que habitan las comunidades y los individuos, que tiene que ver con procesos que explican la intensidad de la relación entre huésped y parásito, que explican la construcción de algún grado de inmunidad frente a Plasmodium spp. y con aspectos que modulan dicha inmunidad, y que determinan el tipo de infección y su duración.

En este sentido, el pasado social de la comunidad, de sus integrantes y de sus relaciones sociales y culturales, permitirán comprender cómo ha sido la construcción histórica de la susceptibilidad de los individuos y las comunidades. Aspectos como los antecedentes laborales de los integrantes, determinados por la historia misma de los procesos productivos de la zona palúdica; sus ubicaciones y sus condiciones; las lógicas productivas y sus relaciones alrededor del trabajo de acuerdo con la clase social, el género y la etnia; la historia de los movimientos migratorios de las comunidades, pasados y recientes, voluntarios u obligados, por razones de tipo económico, cultural o político; y la historia de las comunidades como tal, incluyendo sus orígenes, la etnia o etnias que las conforman y aspectos de su historia cultural, permitirán ayudar a comprender de forma general la presencia y frecuencia de entidades que aumentan 0 disminuyen la sensibilidad a la infección, tales como las hemoglobinopatías, el grado de inmunidad adquirida frente a la enfermedad, la desnutrición crónica, las deficiencias inmunológicas, entre otras, así como la duración y el grado de la infección, la intensidad de la parasitemia y de la gametocitemia,

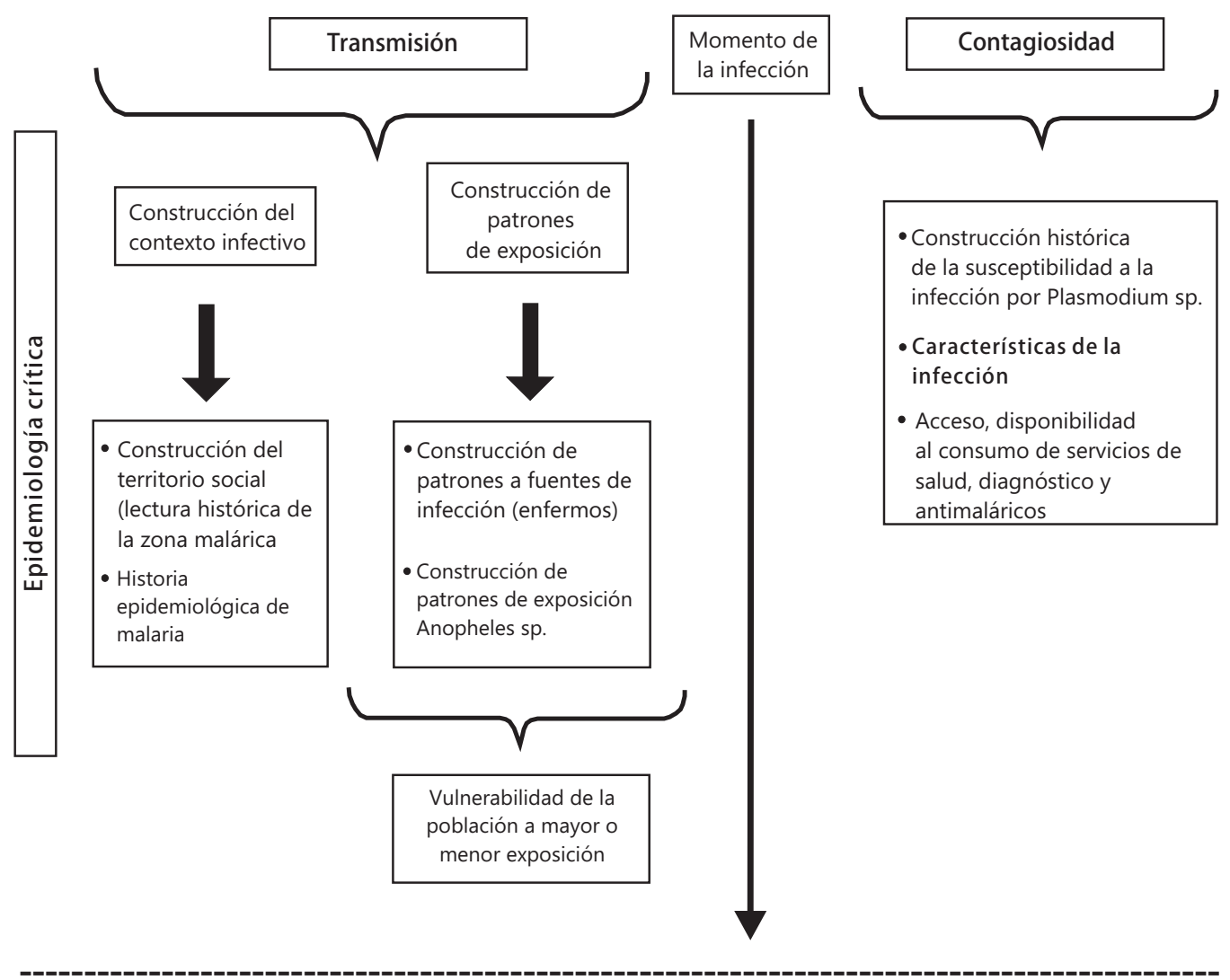

Proceso epidemiológico de la malaria

Basado en: Breilh J. The dialogue of ecology \& epidemiology to deal with complexity and inequity. Honolulu: Working Meeting On Emerging Infectious Disease and Socio-Ecological Systems. Imin International Conference Center, U. of Hawaii, March 9th, 2005.

Figura 1. Reescritura de las categorías explicativas de los procesos infecciosos transmisibles: caso malaria. 
y la frecuencia de procesos infecciosos silentes como las parasitemias asintomáticas o la infección placentaria.

Para finalizar, los procesos directamente involucrados con el consumo de servicios de salud, tanto de servicios generales como los específicamente utilizados en el caso de malaria, ayudarán a comprender la duración de la infección que es determinada socialmente. Para vislumbrar estos aspectos, se debe indagar por aspectos como el tipo de acceso y las barreras de tipo social, económico y cultural a los servicios de salud, el tipo de acceso a acciones colectivas para enfrentar la malaria, el tipo de consumo de antipalúdicos, y la disponibilidad de métodos diagnósticos y tratamientos adecuados, entre otros.

En la figura 1 se esquematiza, en términos generales, los nuevos conceptos de estas categorías desde la epidemiología crítica para el caso de malaria, cuyo contenido surge de la lectura histórica y social de la determinación de la salud y sus elementos explicativos sustanciales. Esta esquematización utilizó el replanteamiento hecho por Jaime Breilh sobre las categorías de exposición y sensibilidad para el caso de agrotóxicos en Ecuador $(25,26)$ y se basó en un esquema previo de este mismo autor presentado en Working Meeting on Emerging Infectious Disease and Socio-Ecological Systems, en Honolulú en el 2005.

\section{Reflexión final}

La gran mayoría de herramientas dirigidas al control de la malaria, han sido diseñadas bajo enfoques que homogenizan la realidad y que ubica la explicación de la salud-enfermedad en los individuos, sin considerar las dinámicas colectivas y lo heterogéneo del espacio-tiempo.

Como lo han señalado algunos autores de la corriente latinoamericana de la medicina social, no repensar las relaciones, no mirar ni comprender los enfoques, por la consideración errónea de que dichas cuestiones pertenecen más al ámbito de la filosofía que de la ciencia, consideraciones que se acrecientan más en este tiempo de gran aceleración y de lógica social eficiente, no ha permitido observar las especificidades paradigmáticas ni el análisis crítico de las acciones emprendidas sobre dicho objeto (4).

Esto hasucedido casi siempre de forma inconsciente para el investigador del área de la salud y esa inconsciencia, sumada a una cierta falta de interés en el tema epistemológico, perpetúa la mirada acrítica y sólo desde el plano de la técnica, tanto del estudio de la salud como de las propuestas dirigidas a mejorarla, es decir, sin que las acciones que generan permitan ninguna transformación de fondo.

Por tanto, comparto con muchos otros la urgencia de "reoxigenar" la academia y la ciencia en salud, con el debate profundo y crítico sobre el pensamiento y su construcción, y con el diálogo honesto y abierto entre las disciplinas, con el fin de configurar la masa crítica necesaria para emprender la reflexión integral de los procesos de salud o enfermedad y el diseño de alternativas para generar procesos de cambio y transformación social dirigidos al bienestar colectivo.

\section{Agradecimientos}

El autor quiere expresar el enorme agradecimiento a los miembros del Área de Salud de la Universidad Andina Simón Bolívar, sede Ecuador, por su apoyo $y$, en particular, a Jaime Breilh, ya que este texto es producto de la pasantía doctoral realizada por el autor bajo su tutoría. Asimismo, agradece su enorme gentileza por autorizar la adaptación de la matriz de procesos críticos de la determinación social y la figura: reescritura de las categorías explicativas de los procesos infecciosos transmisibles, con motivo de esta publicación.

Igualmente, extiende los agradecimientos a Silvia Blair del Grupo Malaria de la Universidad de Antioquia y a Mario Hernández del Doctorado Interfacultades en Salud Pública de la Universidad Nacional, por la lectura crítica y comentarios al texto.

\section{Conflicto de intereses}

El autor manifiesta que no existe conflicto de intereses.

\section{Financiación}

La escritura de este texto fue financiado por el Grupo Malaria de la Universidad de Antioquia y por Colciencias en el marco del proyecto Malaria Gestacional y Congénita en Urabá, código 111504-17041.

\section{Referencias}

1. Breilh J. Epidemiología crítica. Ciencia emancipadora e interculturalidad. Buenos Aires: Lugar Editorial; 2003.

2. Caponi S. The tropics, microbes, and vectors. Hist Cienc Saude Manguinhos. 2002;9(Suppl.):111-38.

3. World Health Organization. World malaria report 2008. Fecha de consulta: febrero de 2009. Disponible en: http:// malaria.who.int/wmr2008/malaria2008.pdf. 
4. Byass P. Making sense of long-term changes in malaria Lancet. 2008;372:1523-5.

5. Almeida-Filho N, Paim-Silva J. La crisis de la salud pública y el movimiento de salud colectiva en Latinoamérica. Cuadernos Médicos Sociales. 1999;75:5-30.

6. Waitzkin $\mathbf{H}$, Iriart $\mathbf{C}$, Estrada A, Lamadrid S. Social medicine then and now: lessons from Latin America. Am J Public Health. 2001;91:1592-601.

7. Almeida Filho N. For a general theory of health: preliminary epistemological and anthropological notes. Cad Saude Publica. 2001;17:753-70.

8. Iriart C, Waitzkin H, Breilh J, Estrada A, Merhy EE. Latin American social medicine: contributions and challenges. Rev Panam Salud Pública. 2002;12:128-36.

9. Samaja J. Desafíos a la epidemiología (pasos para una epidemiología "Miltoniana"). Rev Bras Epidemiol. 2005;6:105-20.

10. Granda E. A qué llamamos salud colectiva, hoy. Rev Cub Salud Pública. 2004;30. Fecha de consulta: mayo de 2008. Disponible en: http://scielo.sld.cu/scielo.php?script=sci_ arttext\&pid=S0864-34662004000200009.

11. Almeida-Filho N. La ciencia tímida. Ensayos de deconstrucción de la epidemiología. Buenos Aires: Lugar Editorial; 2000

12. Breilh J. Epidemiología, economía, medicina y política. México D.F.: Distribuciones Fontamara; 1986.

13. Franco $\mathbf{S}$. Anotaciones preliminares para una historia de la causalidad en medicina. En: Nuño J, Bruni B, Franco S. Filosofía en la medicina. Valencia-Venezuela: Universidad de Carabobo, Ediciones del Rectorado; 1993. p. 49-84.

14. Laurell AC. Sobre la concepción biológica y social del proceso salud enfermedad. En: Rodríguez MA. Lo biológico y lo social, su articulación en la formación del personal de salud. Washington D.C.: Organización Panamericana de la Salud; 1994. p. 1-12.

15. García JC. Juan César García entrevista a Juan César García. En: Nunes E. Ciencias sociales y salud en América Latina. Tendencias y perspectivas. Washington D.C.: Organización Panamericana de la Salud; 1986.
16. Lalonde M. A new perspective on the health of Canadians. A working document. Ottawa: Department of Supply and Services; 1974. Fecha de consulta: septiembre de 2009. Disponible en: http://www.hc-sc.gc.ca/hcs-sss/alt formats/ hpb-dgps/pdf/pubs/1974-lalonde/lalonde-eng.pdf.

17. World Health Organization. Declaration of Alma Ata. Fecha de consulta: septiembre de 2009. Disponible en: http://www. who.int/hpr/NPH/docs/declaration_almaata.pdf

18. Susser M, Susser E. Choosing a future for epidemiology II. From black box to Chinese boxes and eco-epidemiology. Am J Public Health. 1996;86:674-7.

19. Navarro V. What we mean by social determinants of health. Int J Health Serv. 2009;39:423-41.

20. Santos M. La naturaleza del espacio. Técnica y tiempo. Razón y emoción. Barcelona: Editorial Ariel Geografía, S.A.; 2000

21. Santos M. Saúde e ambiente no processo de desenvolvimento. Cien Saude Colet. 2003:8:309-14.

22. Sabroza PC, Toledo LM, Osanai CH. A organização do espaço e os processos endêmico-epidêmicos. En: Leal MC, Sabroza PC, Rodrigues RH, Buis PM, editores. Saúde, ambiente e desenvolvimento. São Paulo: Hucitec; 1992. p. 57-77.

23. Ríos-Velásquez CM, Codeço CT, Honório NA, Sabroza PS, Moresco M, Cunha ICL, et al. Distribution of dengue vectors in neighborhoods with different urbanization types of Manaus, state of Amazonas, Brazil. Mem Inst Oswaldo Cruz. 2007;102:617-23.

24. Halloran E. Concepts of infectious disease epidemiology. En: Rothman K, Greenland S. Modern epidemiology. Philadelphia: Lippincott-Raven; 1998. p. 529-54.

25. Breilh J. Nuevo modelo de acumulación y agroindustria: implicaciones ecológicas y epidemiológicas de la floricultura en Ecuador. Cien Saude Colet. 2007;12:91-104.

26. Breilh J. Innovación metodológica, construcción intercultural del conocimiento y empoderamiento: investigación e intervención de una región agroindustrial floricultura. International Development Research Center. Fecha de consulta: mayo de 2008. Disponible en: http://www.idrc.ca/ uploads/user-S/11576346761breilh_presentacion.pdf. 